\title{
Combining harmonic generation and laser chirping to achieve high spectral density in Compton sources
}

\author{
Balša Terzić* \\ Department of Physics, Old Dominion University, Norfolk, Virginia 23529, USA \\ and Center for Accelerator Science, Old Dominion University, Norfolk, Virginia 23529, USA \\ Cody Reeves \\ Department of Engineering Sciences and Applied Mathematics, Northwestern University, \\ Evanston, Illinois 60208, USA and Jefferson Lab, Newport News, Virginia 23606, USA \\ Geoffrey A. Krafft \\ Department of Physics, Old Dominion University, Norfolk, Virginia 23529, USA; \\ Center for Accelerator Science, Old Dominion University, Norfolk, Virginia 23529, USA \\ and Jefferson Lab, Newport News, Virginia 23606, USA
}

(Received 16 September 2015; published 25 April 2016)

\begin{abstract}
Recently various laser-chirping schemes have been investigated with the goal of reducing or eliminating ponderomotive line broadening in Compton or Thomson scattering occurring at high laser intensities. As a next level of detail in the spectrum calculations, we have calculated the line smoothing and broadening expected due to incident beam energy spread within a one-dimensional plane wave model for the incident laser pulse, both for compensated (chirped) and unchirped cases. The scattered compensated distributions are treatable analytically within three models for the envelope of the incident laser pulses: Gaussian, Lorentzian, or hyperbolic secant. We use the new results to demonstrate that the laser chirping in Compton sources at high laser intensities: (i) enables the use of higher order harmonics, thereby reducing the required electron beam energies; and (ii) increases the photon yield in a small frequency band beyond that possible with the fundamental without chirping. This combination of chirping and higher harmonics can lead to substantial savings in the design, construction and operational costs of the new Compton sources. This is of particular importance to the widely popular laser-plasma accelerator based Compton sources, as the improvement in their beam quality enters the regime where chirping is most effective.
\end{abstract}

DOI: 10.1103/PhysRevAccelBeams.19.044403

\section{INTRODUCTION}

Compton or Thomson sources are increasingly being considered as potential sources of high energy photons [1-4]. A principal attraction of such sources is the narrow bandwidth generated in the output radiation. As a narrow bandwidth is desired, it is important to understand the sources of line width [5] in the scattered radiation and to eliminate them to the extent possible. Ghebregziabher et al. [6] were first to report compensating the local value of the frequency in the incident laser pulse against the ponderomotive longitudinal velocity change. Their chirping prescription was near-perfect, in the sense that the subsidiary peaks in the corrected spectrum were still not entirely suppressed. Analytic expressions for the proper frequency modulation (FM) to achieve perfect compensation for any

\footnotetext{
*bterzic@ odu.edu

Published by the American Physical Society under the terms of the Creative Commons Attribution 3.0 License. Further distribution of this work must maintain attribution to the author(s) and the published article's title, journal citation, and DOI.
}

laser pulse shape were later reported in [7] (hereafter TDHK2014). This was followed up by similar derivation of the proper frequency modulation for the circular polarization [8] (as opposed to linear as in TDHK2014) and when electron recoil and spin are taken into consideration [9].

It has been understood for many years in the freeelectron laser (FEL) community [10-12] that harmonic generation can provide a path to a given radiation wavelength with a smaller electron energy than is needed for fundamental emission, or that harmonic generation provides access to shorter wavelengths for a given electron beam energy. This idea, as applied to Compton sources, suffers from the fact that whenever the field strength is low enough that ponderomotive broadening is negligible, emission into the harmonics falls off rapidly with harmonic number. On the other hand, if the field strength is large enough for substantial harmonic emission, ponderomotive broadening limits the spectral density of the emission, increasingly at high harmonic number. The main motivation for our present work is to point out how important it is that the new chirping prescriptions remove ponderomotive broadening from the harmonics, allowing high emitted 
spectral density in the harmonics at high field strength. Combining laser chirping and the higher harmonics is particularly beneficial for the Compton sources based on the laser plasma accelerators, as their improved beam energy spread enters the range in which the chirping is most effective [13-16]. Experimental validation of the new chirping prescriptions should be possible.

In this paper, we report on calculations completed using a classical formalism valid in the Thomson limit [17]. This approximation is invalid in situations with highest field strengths where emission of multiple photons per electron can occur and in Compton sources where x-rays are produced from beams of energy greater than $100 \mathrm{MeV}$, where Compton recoil is a concern $[18,19]$. However, many Compton sources reside in this domain. The field strengths and laser frequencies of calculations presented in this paper are safely within the classical Thomson regime. It is known that similar chirping prescriptions can be developed for scattering including recoil [9]. It is a highly interesting question, still open to the best of our knowledge, whether emission of multiple photons per electron at the highest field strengths may be similarly controlled.

The paper is organized as follows. In Sec. II, we calculate spectra in both the compensated and uncompensated cases in regimes where the normalized vector potential is of order 1 , i.e., in a parameter regime where significant ponderomotive broadening is expected. In Sec. III, we then use the new results to demonstrate that the laser chirping in Compton sources enables them to retain the narrowband radiation and increase the photon yield in all harmonics at high laser intensities. The increase in the photon yield enables the efficient use of higher order harmonics, which greatly reduces the strain on the electron beam source. Finally, we discuss the importance of the new results and conclude in Sec. IV.

\section{ANALYTIC EXPRESSIONS FOR THE ENERGY SPECTRA}

Ponderomotive line broadening is due to the variable redshifting of the emitted radiation because the longitudinal velocity of the electrons changes within the incident laser pulse. Here, as in our previous efforts, calculations are completed using the formalism developed in K2004 for Thomson back scattering. In this reference the far-field spectral distribution of photons Thomson-scattered by a single electron are derived. The incident laser pulse is described by a plane wave. The treatment is fully relativistic and includes the classical electron motion without approximation. We assume a linearly polarized incident plane wave described by a single component for the normalized vector potential $\tilde{A}(\xi)=e A(\xi) / m c=$ $a(\xi) \cos (2 \pi \xi f(\xi) / \lambda)$ [20] where $a(\xi)$ describes the envelope of the oscillation, $\xi=z+c t$ is the coordinate along the laser pulse, $f(\xi)$ specifies the laser FM, and $\lambda$ is a normalizing wavelength for the incident plane wave.
Expressions for backscattered radiation spectra in high intensity Compton sources emitted by a beam with an energy spread are derived from the corresponding equations for scattering off a single electron. K2004 derives expressions for the constant-frequency laser pulses, while TDHK2014 provides the spectra for FM laser pulses.

Both derivations for the backscattered radiation spectrum $\left(d^{2} E / d \omega d \Omega\right)_{\text {beam }}$ start with the integral

$$
\left[\frac{d^{2} E(\omega)}{d \omega d \Omega}\right]_{\text {beam }}=\int_{1}^{\infty} N(\gamma) \frac{d^{2} E(\gamma, \omega)}{d \omega d \Omega} d \gamma,
$$

where $\omega$ is the frequency of the scattered radiation, $\Omega$ is the solid angle of the radiation, $\gamma$ is the relativistic factor, $N(\gamma)$ is the beam's energy distribution and $d^{2} E / d \omega d \Omega$ is the energy emitted per unit frequency per unit solid angle produced by a single electron. The single-electron scattering spectrum can be expressed as in TDHK2014

$$
\frac{d^{2} E(\gamma, \omega)}{d \omega d \Omega}=\left[\frac{d^{2} E(\gamma)}{d \omega d \Omega}\right]_{n}\left[\frac{\omega}{\omega_{0}(\gamma)}\right]^{2}\left|\frac{(1+\beta) \gamma D_{x}(\gamma, \omega)}{\lambda}\right|^{2},
$$

where $\omega_{0}(\gamma)=(1+\beta)^{2} \gamma^{2} 2 \pi c / \lambda$ is the normalizing frequency, $\left[d^{2} E(\gamma) / d \omega d \Omega\right]_{n}=(1+\beta)^{2} \gamma r_{e} E_{\text {beam }} / c$ is the normalization factor and $c$ the speed of light. We recast the frequency content form $D_{x}$ from their Eq. (7)

$$
\begin{aligned}
\tilde{D}_{x} & {\left[\frac{\omega}{\omega_{0}(\gamma)}\right] } \\
& \equiv \frac{(1+\beta) \gamma D_{x}(\gamma, \omega)}{\lambda} \\
& =\frac{1}{2} \int_{-\infty}^{\infty} a(\xi) \exp \left\{-2 \pi i\left[\xi f(\xi)+\frac{\omega}{\omega_{0}(\gamma)} \tilde{Z}(\xi)\right]\right\} d \xi,
\end{aligned}
$$

where $\tilde{Z}(\xi)=\xi+\int_{-\infty}^{\xi} \tilde{A}^{2}\left(\xi^{\prime}\right) d \xi^{\prime}$ and the FM function

$$
f(\xi)=\frac{1}{1+a(0)^{2} / 2}\left[1+\frac{\int_{0}^{\xi} a\left(\xi^{\prime}\right)^{2} d \xi^{\prime}}{2 \xi}\right] .
$$

Therefore, the beam energy only scales the spectrum in both frequency (independent variable) and amplitude (dependent variable):

$$
\frac{d^{2} E(\gamma, \omega)}{d \omega d \Omega}=\left[\frac{d^{2} E(\gamma)}{d \omega d \Omega}\right]_{n}\left[\frac{\omega}{\omega_{0}(\gamma)}\right]^{2}\left|\tilde{D}_{x}\left[\frac{\omega}{\omega_{0}(\gamma)}\right]\right|^{2} .
$$

This means that for a set of laser pulse parametersamplitude $a_{0}$ of the normalized vector potential, envelope shape, wavelength $\lambda$, width $\sigma$-it is necessary to compute only one scale-free spectrum, $\tilde{D}_{x}\left(\omega / \omega_{0}\right)$, generated from the pulse's backscattering off a single electron, as in K2004 and TDHK2014. The computation of Eq. (1) is then 
reduced to integration of that scale-free spectrum, properly shifted and scaled. This treatment applies to both constant frequency scattering of K2004 and FM scattering in TDHK2014, and to all harmonics [9].

Figure 2(b) in TDHK2014 compares the frequency bandwidth (Fourier transform) of the incoming laser pulse and the backscattered radiation after the FM and demonstrates a perfect agreement of the two in bandwidth and a near-perfect agreement (to within a couple of percent) in the maximum amplitude. Therefore, to first order, one can approximate the first harmonic of the backscattered radiation spectrum for a properly FM laser pulse by its Fourier transform:

$$
\tilde{D}_{x}\left(\frac{\omega}{\omega_{0}(\gamma)}\right)=\frac{1}{2} \mathcal{F}\{a(\xi)\}\left(\frac{\frac{\omega}{\omega_{0}(\gamma)}-\tilde{w}_{f}}{\tilde{w}_{f}}\right),
$$

where the scaling of the peak width by $\tilde{w}_{f}=1 /\left(1+a_{0}^{2} / 2\right)$ is due to the first-order expansion of $\tilde{Z}(\xi)$ around $\xi=0$ in Eq. (3). For the three laser pulse shapes considered hereGaussian, Lorentzian and hyperbolic secant, defined in Table I-they are

$$
\begin{aligned}
& \tilde{D}_{x, G}\left(\frac{\omega}{\omega_{0}}\right)=a_{0} \sigma \sqrt{\frac{\pi}{2}} \exp \left[-\frac{\left(\frac{\omega}{\omega_{0}}-\tilde{w}_{f}\right)^{2}}{2 \bar{\Sigma}^{2}}\right], \\
& \tilde{D}_{x, L}\left(\frac{\omega}{\omega_{0}}\right)=\frac{a_{0} \pi \sigma}{4} \exp \left(-\frac{\left|\frac{\omega}{\omega_{0}}-\tilde{w}_{f}\right|}{2 \bar{\Sigma}}\right), \\
& \tilde{D}_{x, S}\left(\frac{\omega}{\omega_{0}}\right)=\frac{a_{0} \pi}{2 \sigma} \operatorname{sech}\left[\frac{\pi^{2}}{\bar{\sigma}}\left(\frac{\omega}{\omega_{0}}-\tilde{w}_{f}\right)\right],
\end{aligned}
$$

where $\Sigma=1 / 2 \pi \sigma, \bar{\Sigma}=\Sigma \tilde{w}_{f}$ and $\bar{\sigma}=\sigma \tilde{w}_{f}$. These are achieved by FM given in TDHK2014 and our Eq. (4), which for the three laser pulse shapes are

$$
\begin{gathered}
f_{G}(\bar{\xi})=\tilde{w}_{f}\left[1+\frac{\sqrt{\pi} \sigma a_{0}^{2}}{4 \bar{\xi}} \operatorname{erf}(\bar{\xi} / \sigma)\right], \\
f_{L}(\bar{\xi})=\tilde{w}_{f}\left[1+\frac{\sqrt{\sigma} a_{0}^{2}}{4 \sqrt{2} \bar{\xi}}\left(\frac{\sqrt{2 \sigma} \bar{\xi}}{\sigma+2 \bar{\xi}^{2}}+\tan ^{-1} \frac{\sqrt{2} \bar{\xi}}{\sqrt{\sigma}}\right)\right], \\
f_{S}(\bar{\xi})=\tilde{w}_{f}\left[1+\frac{a_{0}^{2}}{2 \bar{\xi} \sigma} \tanh (\sigma \bar{\xi})\right],
\end{gathered}
$$

with $\bar{\xi}=\xi / \lambda$.

TABLE I. Laser pulse shapes considered here.

\begin{tabular}{ll}
\hline \hline Gaussian & $a_{G}(\xi)=a_{0} \exp \left[-\xi^{2} /\left(2(\sigma \lambda)^{2}\right)\right]$ \\
\hline Lorentzian & $a_{L}(\xi)=a_{0} \sigma /\left[(\xi / \lambda)^{2}+\sigma\right]$ \\
Hyperbolic secant & $a_{S}(\xi)=a_{0} \operatorname{sech}(\sigma \xi / \lambda)$ \\
\hline
\end{tabular}

Obtaining an analytic approximation for the shape of the higher-order harmonics in the FM spectrum requires a derivation similar to that in Brau [21]. After substituting Eq. (4) into Eq. (3), integrating by parts, using $\exp (i \alpha \sin \theta)=\sum_{n=-\infty}^{\infty} J_{n}(\alpha) \exp (\operatorname{in} \theta)$, where $J_{n}$ is the Bessel function of the $n$th order, and expanding around the stationary phase point, we find the contribution of the $n$th harmonic to the scale-free spectrum $\tilde{D}_{x}=\sum_{n} \tilde{D}_{x}^{n}$

$$
\tilde{D}_{x}^{n}=\int_{0}^{\infty} a(\xi) K_{n}[g(\xi)] \cos \left[2 \pi Z(\xi) \bar{\omega}_{n}\right] d \xi,
$$

with $\quad K_{n}(\alpha)=(-1)^{n}\left[J_{n}(\alpha)-J_{n-1}(\alpha)\right], \quad g(\xi)=$ $a^{2}(\xi)(n-1 / 2) /\left\{2\left[1+1 / 2 a^{2}(\xi)\right]\right\}, \quad \bar{\omega}_{n}=\omega / \omega_{0}-$ $2(n-1 / 2) \tilde{w}_{f} \quad$ and $\quad Z(\xi)=\xi+(1 / 2) \int_{0}^{\xi} a^{2}\left(\xi^{\prime}\right) d \xi^{\prime}$. Examining the $K_{n}(g(\xi))$ term, an implicit function of $\xi$, reveals that the explicit function $\tilde{K}_{n}(\xi)$ is wellapproximated by a Gaussian $\tilde{K}_{n}(\xi) \approx A_{n} \exp \left(-\xi^{2} / 2 \Sigma_{n}\right)$ for $n>1$ and a shifted Gaussian $\tilde{K}_{1}(\xi) \approx$ $1+A_{1} \exp \left(-\xi^{2} / 2 \Sigma_{1}\right)$ for $n=1$ [because $J_{0}(0)=1$, $J_{n}(0)=0$ for $n>1$; only $K_{1}$ contains $J_{0}$, where $\quad A_{1}=K_{1}[g(0)]-1, \quad A_{n}=K_{n}[g(0)], \quad \Sigma_{1}=$ $\sqrt{-B_{1}^{2} / 2 \log \left[\left(B_{1}-1\right) / A_{1}\right]}, \quad \Sigma_{n}=\sqrt{-B_{n}^{2} / 2 \log \left(B_{n} / A_{n}\right)}$ $B_{n}=K_{n}[g(\sigma)]$. Keeping only the linear term in $Z(\xi)$, we obtain analytic approximation for all harmonics:

$$
\begin{array}{r}
\tilde{D}_{x}^{1}=\frac{1}{2} \mathcal{F}\left\{a(\xi)\left(1+A_{1} \exp \left[-\frac{\xi^{2}}{2 \Sigma_{n}^{2}}\right]\right)\right\}\left(\frac{\bar{\omega}_{1}}{\tilde{w}_{f}}\right), \\
\tilde{D}_{x}^{n}=\frac{1}{2} \mathcal{F}\left\{a(\xi) A_{n} \exp \left[-\frac{\xi^{2}}{2 \Sigma_{n}^{2}}\right]\right\}\left(\frac{\bar{\omega}_{n}}{\tilde{w}_{f}}\right) .
\end{array}
$$

If one neglects a correction due to the small parameter $A_{1}$, the previous result for the leading-order harmonic in Eq. (6) is recovered. An excellent agreement between this approximation and the exact scale-free solution is shown in Fig. 1. For the higher-order harmonics, only Gaussian pulse yields an analytic approximation, which is shown in Fig. 2. The agreement is nearly perfect. The Eq. (10) suggests, and Fig. 3 corroborates, that only for the Gaussian laser pulse is the shape of all the harmonics the same (Gaussian in this case). The higher-order harmonics of the Lorentzian and hyperbolic secant pulse are different from their respective first harmonics.

The height of the harmonics is computed from Eq. (10) by substituting $\int_{-\infty}^{\infty} d \xi$ for $\mathcal{F}\{\}$ :

$$
\begin{aligned}
\left|\tilde{D}_{x}^{1, n}\right|_{\max , G} & =a_{0} \sqrt{\frac{\pi}{2}}\left(\sigma+A_{1} s_{G}^{1}, A_{n} s_{G}^{n}\right), \\
\left|\tilde{D}_{x}^{1, n}\right|_{\max , L} & =a_{0}\left(\pi \sqrt{\sigma}+A_{1} s_{L}^{1}, A_{n} s_{L}^{n}\right),
\end{aligned}
$$




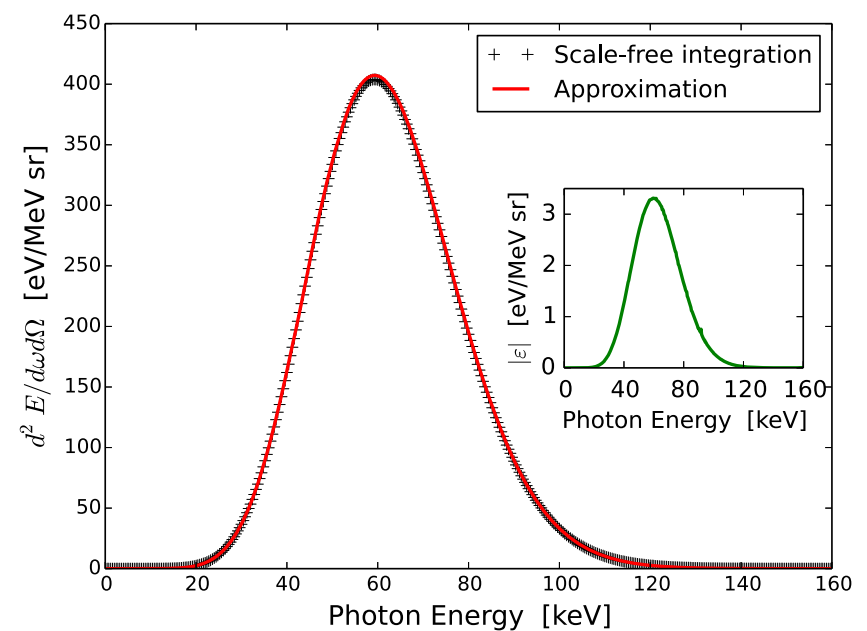

FIG. 1. Integration of a single scale-free spectrum $\tilde{D}_{x}$ and an electron beam with a $34 \%$ energy spread versus the approximation from Eq. (13a) (red line). The inset shows the absolute difference $\varepsilon$ between the two results. Spectra are computed from scattering a FM Gaussian laser pulse with $\lambda=800 \mathrm{~nm}, a_{0}=$ 0.587 off a Gaussian electron beam with $Q=100 \mathrm{pC}$ and $E_{\text {beam }}=51.1 \mathrm{MeV}$.

where $s_{G}^{n}=\sigma \Sigma_{n} / \sqrt{\Sigma_{n}^{2}+\sigma^{2}}, \quad s_{L}^{n}=(\pi / 2 \sqrt{\sigma}) \exp \left(\beta_{n}^{2}\right) \times$ $\left[1-\operatorname{erf}\left(\beta_{n}\right)\right]$ and $\beta_{n}=\sqrt{\sigma / 2 \Sigma_{n}^{2}}$. The width of the harmonics for the Gaussian pulse are

$$
W_{G}^{1} \approx \frac{\tilde{w}_{f}}{2 \pi \sigma}, \quad W_{G}^{n}=\frac{\tilde{w}_{f}}{2 \pi s_{G}^{n}} .
$$

For FWHM in eV, these are multiplied by $2.35 \hbar \omega_{0}$.

For the Gaussian electron beam distribution and the case with FM, substituting Eqs. (7) into Eq. (5) and the integral

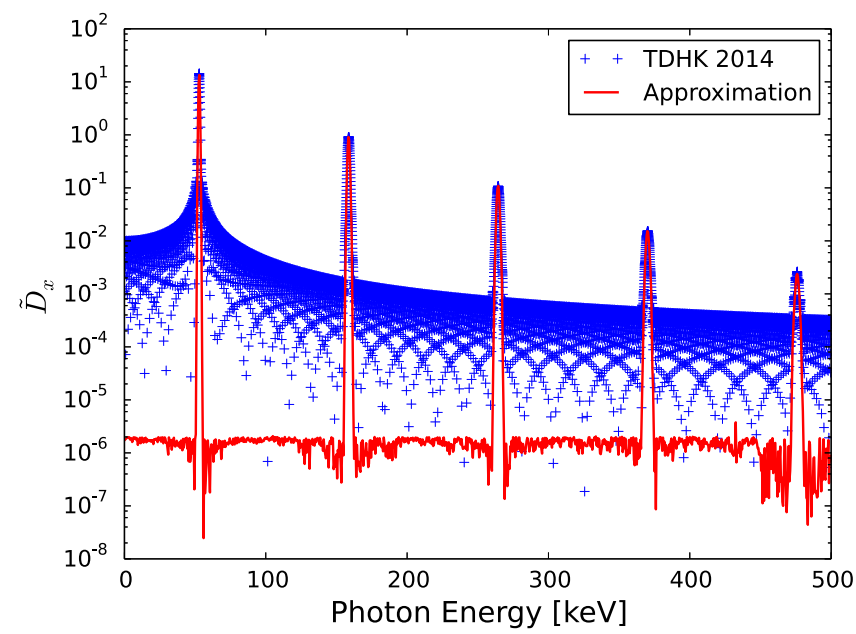

FIG. 2. Single-electron scattering approximation in Eq. (10) against the exact solution of TDHK2014. Spectra are computed from scattering a FM Gaussian laser pulse with $\lambda=800 \mathrm{~nm}$, $a_{0}=0.587$ off a Gaussian electron beam with $Q=100 \mathrm{pC}$ and $E_{\text {beam }}=51.1 \mathrm{MeV}$.
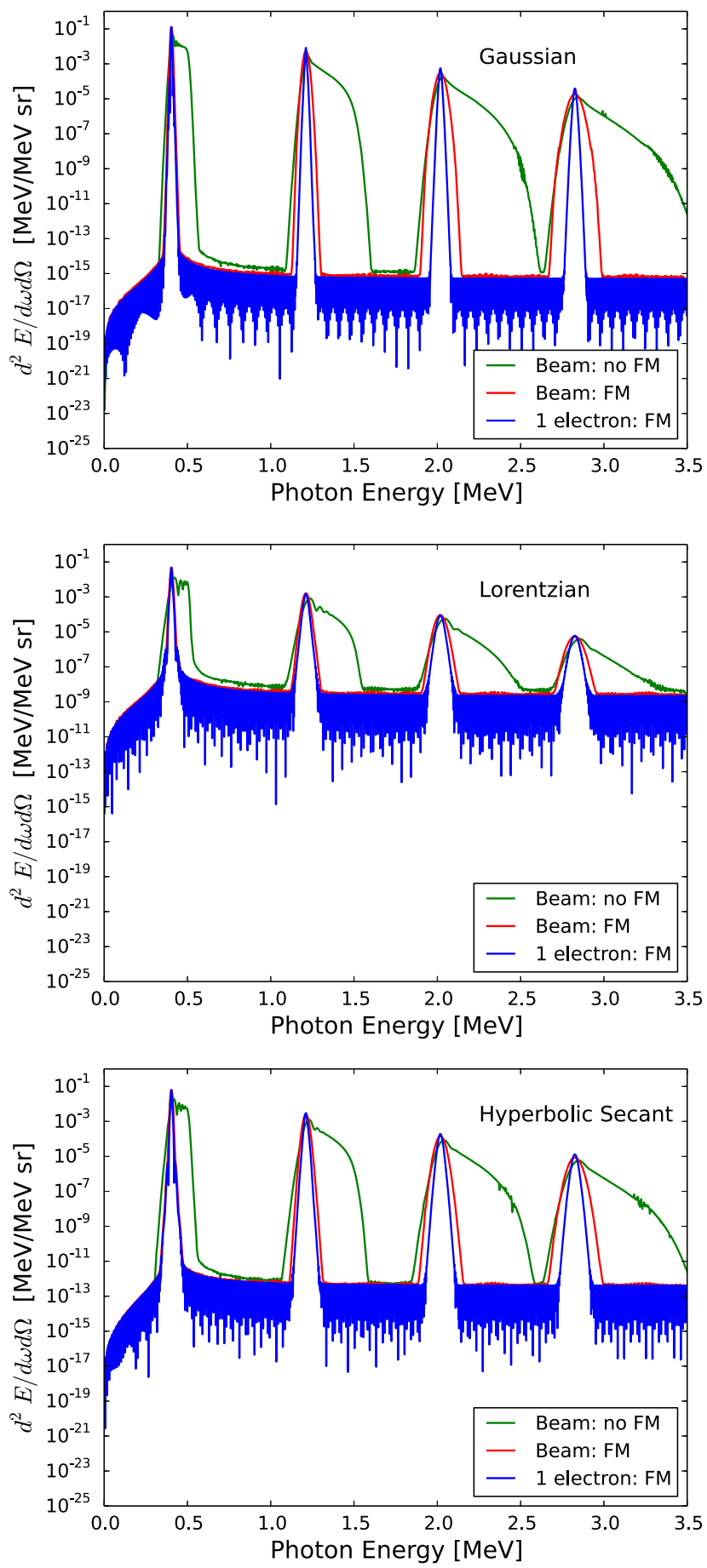

FIG. 3. Backscattered radiation of a laser pulse with $\lambda=1 \mu \mathrm{m}$, $a_{0}=0.707$ off a Gaussian electron beam with $Q=100 \mathrm{pC}$, $E_{\text {beam }}=163 \mathrm{MeV}$, and $1 \%$ FWHM energy spread: FM (red), non-FM (green), and the single electron FM case (normalized to FM) (blue). Top: Gaussian pulse. Middle: Lorentzian pulse. Bottom: Hyperbolic secant pulse.

in Eq. (1) leads to analytic solutions after invoking a welljustified approximation that the narrowband backscattered radiation off a single electron $d^{2} E(\gamma, \omega) / d \omega d \Omega$ is highly peaked around $\bar{\gamma}(\omega)=\sqrt{\lambda \omega / 2 \pi c \tilde{w}_{f}}$ over which range 
$1 / \gamma^{2}$ can be considered constant. Then the single-electron spectra are well-approximated by a Gaussian, which exactly integrates into analytic expressions for the leadingorder harmonic:

$$
\begin{aligned}
\left(\frac{d^{2} E(\omega)}{d \omega d \Omega}\right)_{\text {beam }, \mathrm{FM}, \mathrm{G}}= & \frac{\operatorname{er}_{e} a_{0}^{2} \sigma^{2} \lambda \tilde{w}_{f}}{4 q_{e} c^{2}} \frac{S_{G}(\omega) \omega}{\sqrt{\tilde{\sigma}_{E}^{2}+S_{G}(\omega)^{2}}} \\
& \left.\times \exp \left\{-\frac{\left[\gamma_{0}-\bar{\gamma}(\omega)\right]^{2}}{2\left[\tilde{\sigma}_{E}^{2}+S_{G}^{2}(\omega)\right]}\right\}, \quad 113 \mathrm{a}\right) \\
\left(\frac{d^{2} E(\omega)}{d \omega d \Omega}\right)_{\text {beam,FM }, \mathrm{L}}= & \frac{\operatorname{Qer}_{e} a_{0}^{2} \sigma^{2} \lambda \tilde{w}_{f} \pi^{3}}{32 \sqrt{2} q_{e} c^{2} \tilde{\sigma}_{E}} \\
& \times\left\{r_{+}\left[1-\operatorname{erf}\left(s_{+}\right)\right]-r_{-} \operatorname{erfi}\left(s_{-}\right)\right\},
\end{aligned}
$$

$$
\begin{aligned}
\left(\frac{d^{2} E(\omega)}{d \omega d \Omega}\right)_{\text {beam }, \mathrm{FM}, \mathrm{S}}= & \frac{\operatorname{Qer}_{e} a_{0}^{2} \lambda \tilde{w}_{f} \pi k}{8 q_{e} c^{2} \sigma^{2}} \frac{S_{S}(\omega) \omega}{\sqrt{\tilde{\sigma}_{E}^{2}+S_{S}(\omega)^{2}}} \\
& \times \exp \left\{-\frac{\left[\gamma_{0}-\bar{\gamma}(\omega)\right]^{2}}{2\left[\tilde{\sigma}_{E}^{2}+S_{S}^{2}(\omega)\right]}\right\},
\end{aligned}
$$

where $\quad S_{G}(\omega)=\bar{\gamma}(\omega) \Sigma /\left(2 \sqrt{2} \tilde{w}_{f}\right), \quad S_{L}(\omega)=\bar{\gamma}(\omega) /$ $\left[\sqrt{2 \pi \tilde{w}_{f}}(2 \sigma)^{1 / 4}\right], \quad S_{S}(\omega)=\bar{\gamma}(\omega) \sigma /\left(2 \sqrt{2} \pi^{2} \tilde{w}_{f}\right), \quad k=$ 1.12841 is the normalization factor, erfi the complex error function $\operatorname{erfi}(x)=i \operatorname{erf}(i x)$ and

$$
\begin{gathered}
r_{ \pm}=\frac{\exp \left[\frac{-\gamma_{0}^{2}}{2\left(\tilde{\sigma}_{E}^{2} \pm S_{L}^{2}\right)} \mp 2 \sqrt{2 \sigma} \pi \tilde{w}_{f}\right]}{\sqrt{\tilde{\sigma}_{E}^{2} \pm S_{L}^{2}}} \\
s_{ \pm}=\frac{\bar{\gamma} \tilde{\sigma}_{E}^{2} \pm S_{L}^{2}\left(\bar{\gamma}-\gamma_{0}\right)}{\sqrt{2} \tilde{\sigma}_{E} S_{L} \sqrt{\tilde{\sigma}_{E}^{2} \pm S_{L}^{2}}} .
\end{gathered}
$$

The solutions for a single-electron scattering are easily recovered when $\tilde{\sigma}_{E} \rightarrow 0$ and $Q=q_{e}$ are substituted. There is an excellent agreement between the analytic approximation Eq. (13a) and the numerical integration of Eq. (1) with a single scale-free spectrum $\tilde{D}_{x}$ given in Eq. (3), as shown in Fig. 1. The accuracy for other laser pulse distributions is similarly high. Clearly, one can confidently use either of these methods for the case with FM-direct numerical integration of the electron distribution with a single scale-free spectrum or the analytic approximationwith the latter being preferred for its simplicity and ease of implementation. However, for the case of constantfrequency laser pulse for which the single-electron scattering $d^{2} E(\gamma, \omega) / d \omega d \Omega$ can only be numerically computed as in $\mathrm{K} 2004$, the integration of a single scale-free spectrum is the sole option.

\section{COMBINING HIGHER HARMONICS AND LASER CHIRPING}

Figure 3 shows the spectrum of the backscattered radiation from a electron beam with a $1 \%$ energy spread, with and without FM, and compares it to the spectrum of a single-electron scattering. It is evident that the FM for all three pulse shapes, computed from TDHK2014 as in Eq. (8), is still quite effective in restoring the narrowband spectrum for all harmonics simultaneously.

The bandwidth of the backscattered radiation - for both the FM and constant-frequency laser pulse-is affected by two components: (1) the intrinsic bandwidth of the singleelectron scattering (which depends on the length of the laser pulse $\sigma$ ); and (2) the energy spread of the electron beam distribution, $\sigma_{E}$. The first component provides the absolute theoretical lower limit of a monochromatic beam $\left(\sigma_{E}=0\right)$. When the electron beam energy spread is small, the bandwidth is dominated by the intrinsic single-electron scattering bandwidth (monochromatic limit $\sigma_{E} \rightarrow 0$ ). When the electron beam energy spread is high, the bandwidth is dominated by the bandwidth of the electron beam, thereby diminishing the effects of the FM. This is illustrated in Fig. 4.

\section{Increasing the photon yield}

Figure 5 illustrates the increase in the maximum photon yield $\left(d^{2} N / d \omega d \Omega\right)^{\max }$ after using FM, defined as

$$
R_{\mathrm{FM}} \equiv \frac{\left(d^{2} N / d \omega d \Omega\right)_{\mathrm{FM}}^{\max }}{\left(d^{2} N / d \omega d \Omega\right)_{\mathrm{nonFM}}^{\max }},
$$

as a function of the amplitude of the normalized vector potential $a_{0}$ and intensity. [The relationship between the two quantities is given by $\left.I=2 \pi^{2} m_{e}^{2} c^{3} a_{0}^{2} /\left(\lambda^{2} e^{2} \mu_{0}\right)\right]$. Here $d^{2} E / d \omega d \Omega=\hbar \omega d^{2} N / d \omega d \Omega$. As discussed above, the smaller the beam energy spread, the larger the improvement in photon yield after using FM.

When employing FM, the restored narrow harmonics peak near $E=4 \gamma^{2} E_{p} n /\left(1+(1 / 2) a_{0}^{2}\right)$, where $E_{p}$ is the energy of the laser pulse and $n$ the order of the harmonic. This means that the same desired radiation can be achieved either by using the first harmonic from a backscattering off an electron beam with the energy $\gamma m_{e} c^{2}$, or the $n$th harmonic from a backscattering off an electron beam with energy $\gamma m_{e} c^{2} / \sqrt{n}$. An example is shown in Fig. 6: at $0.1 \%$ FWHM electron beam energy spread, the maximum photon yield in the third harmonic at $163 \mathrm{MeV}$ equals that of in the first peak in the $282 \mathrm{MeV}$ beam. Such a $1 / \sqrt{n}$ reduction in the required electron beam energy would lead to substantial cost savings in the design, construction and shielding of the electron source. A more detailed dependence of the ratio of the maximum yield of the FM $n$th harmonic produced by an electron beam at energy $E / \sqrt{n}$ and the non-FM first harmonic produced by an electron beam at energy $E$ 

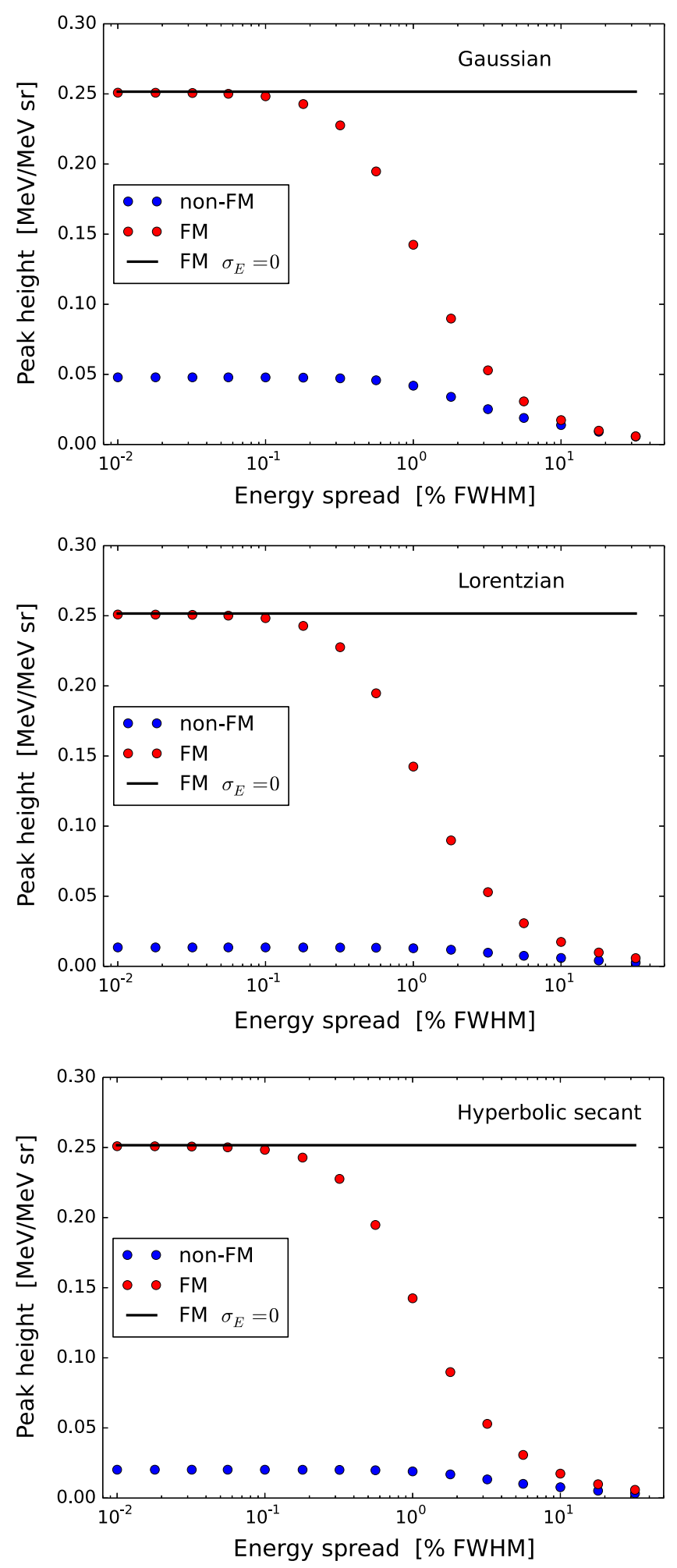

FIG. 4. Maximum amplitude as a function of the energy spread for a laser pulse with $\lambda=1 \mu \mathrm{m}, a_{0}=0.707$ off a Gaussian electron beam with $Q=100 \mathrm{pC}, E_{\text {beam }}=163 \mathrm{MeV}$ without FM (blue dots) and with FM (red dots), along with the theoretical limit for the FM monochromatic $\left(\sigma_{E}=0\right)$ beam from Eq. (13a) (solid black line), computed by scale-free integration. Top: Gaussian laser pulse. Middle: Lorentzian laser pulse. Bottom: Hyperbolic secant laser pulse.

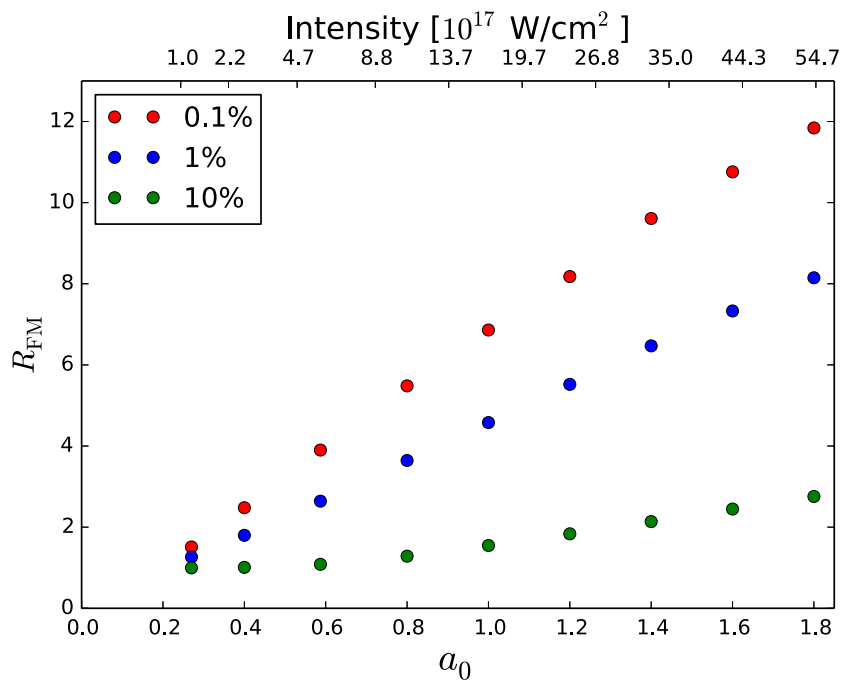

FIG. 5. Ratio of the maximum photon yield between the FM and non-FM laser pulse as a function of the intensity (and the amplitude of the normalized vector potential $a_{0}$ ) for a Gaussian laser pulse with $\lambda=1 \mu \mathrm{m}$ off a Gaussian electron beam with $Q=100 \mathrm{pC}, \quad E_{\text {beam }}=163 \mathrm{MeV}$ and $0.1 \%$ FWHM energy spread (red dots), $1 \%$ (blue) and $10 \%$ (green). The leftmost set of points $\left(a_{0}=0.27\right.$ or $\left.I=10^{17} \mathrm{~W} / \mathrm{cm}^{2}\right)$ corresponds to the current upper limit of operation of the laser-plasma accelerators [16].

$$
R_{1 n} \equiv \frac{\left(d^{2} N / d \omega d \Omega\right)_{\mathrm{FM}, n}^{\max }}{\left(d^{2} N / d \omega d \Omega\right)_{\operatorname{monFM}, 1}^{\max }}
$$

is shown in Fig. 7 for the third and in Fig. 8 for the fifth harmonic. Figure 9 shows that even though the redshift at large amplitudes of the normalized potential

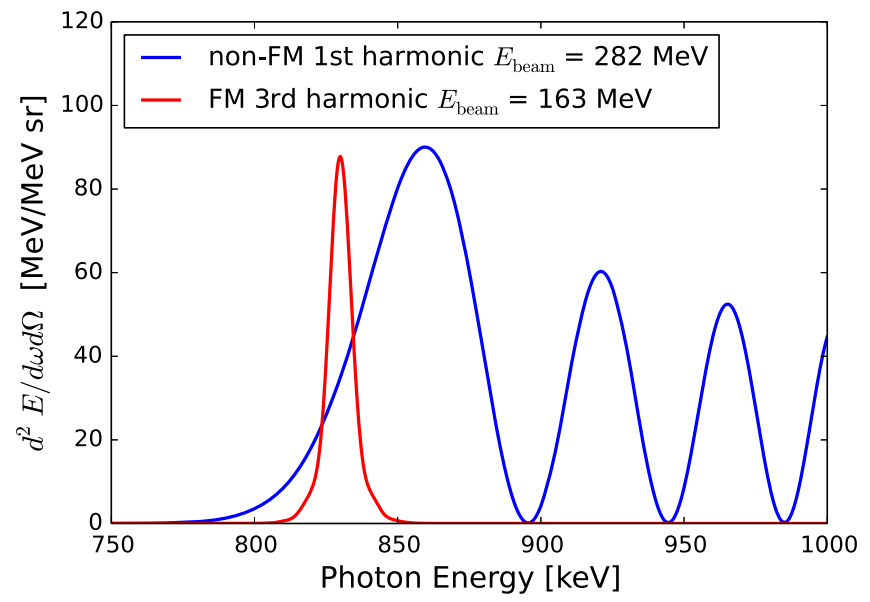

FIG. 6. Backscattered radiation of a laser pulse with $\lambda=1 \mu \mathrm{m}$, $a_{0}=1.6$ off a Gaussian electron beam with $Q=100 \mathrm{pC}, 0.1 \%$ FWHM energy spread: first harmonic from the collision of the non-FM Gaussian pulse and the $282 \mathrm{MeV}$ electron beam (blue); third harmonic from the collision of the FM Gaussian pulse the $163 \mathrm{MeV}$ electron beam (red). 


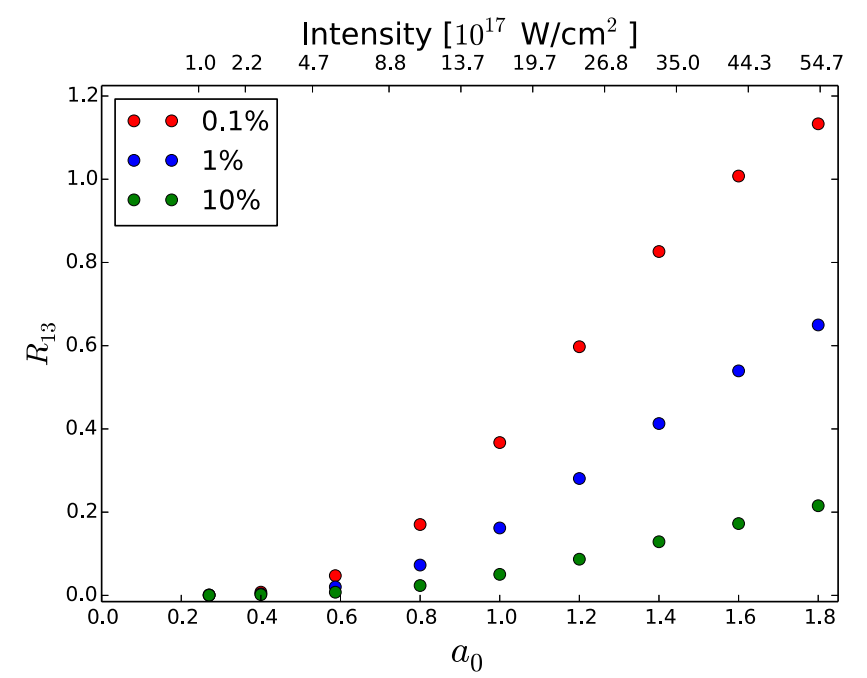

FIG. 7. Ratio of the maximum photon yield between the FM third harmonic from $E_{\text {beam }}=163 \mathrm{MeV}$ and the non-FM first harmonic from $E_{\text {beam }}=282 \mathrm{MeV}$ as a function of the intensity (and the amplitude of the normalized vector potential $a_{0}$ ) for a Gaussian laser pulse with $\lambda=1 \mu \mathrm{m}$ off a Gaussian electron beam with $Q=100 \mathrm{pC}$, and $0.1 \%$ FWHM energy spread (red dots), $1 \%$ (blue) and $10 \%$ (green). The leftmost set of points $\left(a_{0}=0.27\right.$ or $I=10^{17} \mathrm{~W} / \mathrm{cm}^{2}$ ) corresponds to the current upper limit of operation of the laser-plasma accelerators [16].

$a_{0} \gtrsim 10$, the FM spectra still feature clearly delineated peaks. As laser-plasma accelerator performance in the sub$1 \%$ range in energy spread becomes realistic [16], using the FM higher order harmonics is poised to reap substantial benefits.

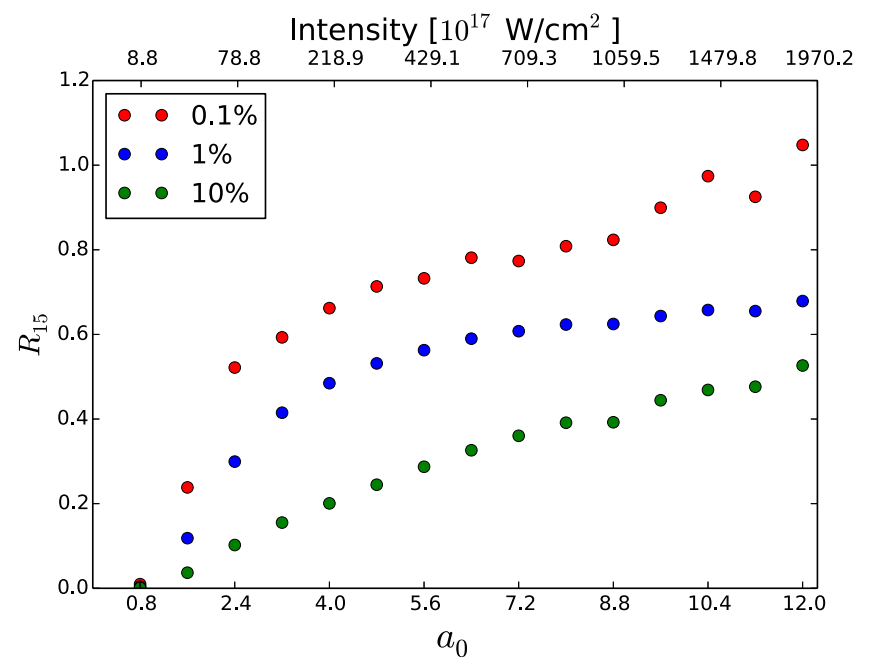

FIG. 8. Ratio of the maximum photon yield between the FM fifth harmonic from $E_{\text {beam }}=126 \mathrm{MeV}$ and the non-FM first harmonic from $E_{\text {beam }}=282 \mathrm{MeV}$ as a function of the intensity (and the amplitude of the normalized vector potential $a_{0}$ ) for a Gaussian laser pulse with $\lambda=1 \mu \mathrm{m}$ off a Gaussian electron beam with $Q=100 \mathrm{pC}$, and $0.1 \%$ FWHM energy spread (red dots), $1 \%$ (blue) and $10 \%$ (green).

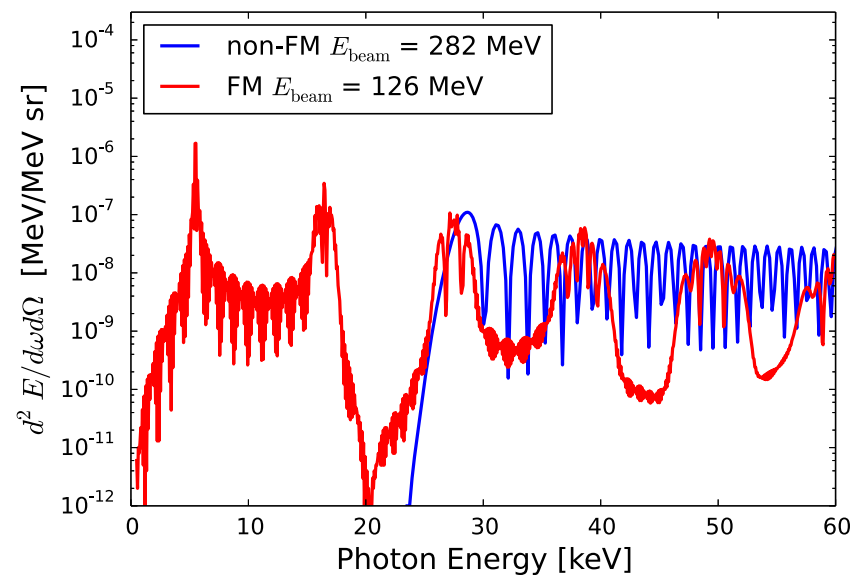

FIG. 9. Backscattered radiation of a non-FM Gaussian laser pulse off a Gaussian electron beam with $E_{\text {beam }}=282 \mathrm{MeV}$ (blue) and a FM Gaussian laser pulse off a Gaussian electron beam with $E_{\text {beam }}=126 \mathrm{MeV}$ (red). $\lambda=1 \mu \mathrm{m}, Q=100 \mathrm{pC}$, and $0.1 \%$ FWHM energy spread.

\section{DISCUSSION AND SUMMARY}

In this paper a novel calculation prescription is used to determine the emission characteristics of the scattered radiation in a Compton back-scatter source. When compensated by laser beam frequency modulation (chirping) the radiation line heights and widths may be accurately computed using a stationary phase argument with crisp functional forms. The calculations accurately account for detuning of the emitted radiation by beam energy spread.

Our calculations suggest the following main conclusion: by combining harmonic generation and frequency modulation (chirping) of the incident laser pulse it should be possible to generate significant fluxes of Compton scattered radiation on the harmonic frequencies. The beam energy spread in laser-plasma acceleration schemes are becoming good enough [16], when they are at the $1 \%$ level, that the energy flux density on the harmonics can even exceed that possible when an unmodulated incident laser pulse is used.

Using higher harmonics at low laser intensities the photon yield is substantially smaller than that in the first harmonic. The yield of the higher harmonics can be improved-both absolute and relatively compared to the first harmonic - by increasing the laser intensity. However, increasing the laser intensity leads to the ponderomotive broadening which effectively erases any advantages thus incurred. Frequency modulation and its perfect restoration of the narrowband emission, becomes crucial: it mitigates the adverse effects of the ponderomotive broadening, allowing one to continue increasing the photon yield.

\section{ACKNOWLEDGMENTS}

This paper is authored by Jefferson Science Associates, LLC under U.S. Department of Energy (DOE) Contract No. DE-AC05-06OR23177. The U.S. Government retains 
a nonexclusive, paid-up, irrevocable, worldwide license to publish or reproduce this manuscript for U.S. Government purposes. C. R. acknowledges the support from the U.S. Department of Energy, Science Undergraduate Laboratory Internship (SULI) program.

[1] G. A. Krafft and G. Priebe, Compton sources of electromagnetic radiation, Rev. Accl. Sci. Tech. 03, 147 (2010).

[2] Z. Huang and R. Ruth, Laser-Electron Storage Ring, Phys. Rev. Lett. 80, 976 (1998). See also www.lynceantech.com.

[3] R. Pantell, G. Soncini, and H. Puthoff, Stimulated photonelectron scattering, IEEE J. Quant. Electr. QE 4, 905 (1968).

[4] F. V. Bunkin, A. E. Kazakov, and M. V. Fedorov, Interaction of intense optical radiation with free electrons (nonrelativistic case), Sov. Phys. Usp. 15, 416 (1973).

[5] F. V. Hartemann and S. S. Wu, Nonlinear Brightness Optimization in Compton Scattering, Phys. Rev. Lett. 111, 044801 (2013).

[6] I. Ghebregziabher, B. A. Shadwick, and D. Umstadter, Spectral bandwidth reduction of Thomson scattered light by pulse chirping, Phys. Rev. Accel. Beams 16, 030705 (2013).

[7] B. Terzić, K. Deitrick, A. Hofler, and G. A. Krafft, NarrowBand Emission in Thomson Sources Operating in the High-Field Regime, Phys. Rev. Lett. 112, 074801 (2014).

[8] S. G. Rykovanov, C. G. R. Geddes, C. B. Scrhoeder, E. Esarey, and W. Leemans, Controlling the spectral shape of nonlinear Thomson scattering with proper laser chirping, Phys. Rev. Accel. Beams 19, 030701 (2016).

[9] D. Seipt, S. G. Rykovanov, A. Surzhykov, and S. Fritzsche, Narrowband inverse Compton scattering X-ray sources at high laser intensities, Phys. Rev. A 91, 033402 (2015).

[10] J. M. J. Madey, Relationship between mean radiated energy, mean squared radiated energy and spontaneous power spectrum in a power series expansion of the equations of motion in a free electron laser, Nuovo Cimento 50B, 64 (1979).

[11] W. B. Colson, The nonlinear wave equation for higher harmonics in free-electron lasers, IEEE J. Quantum Electron. 17, 1417 (1981).

[12] S. V. Benson and J. M. J. Madey, Demonstration of harmonic lasing in a free-electron laser, Phys. Rev. A 39, 1579 (1989).

[13] N. D. Powers, I. Ghebregziabher, G. Golovin, C. Liu, S. Chen, S. Banerjee, J. Zhang, and D. P. Umstadter, Quasimonoenergetic and tunable X-rays from a laser-driven Compton light source, Nat. Photonics 8, 28 (2014).

[14] G. Sary et al., Ultrahigh Brilliance Multi-MeV $\gamma$-Ray Beams from Nonlinear Relativistic Thomson Scattering, Phys. Rev. Lett. 113, 224801 (2014).

[15] K. Khernnikov, J. Wenz, A. Buck, J. Xu, M. Heigoldt, L. Veisz, and S. Karsch, Tunable All-Optical Quasimonochromatic Thomson X-Ray Source in the Nonlinear Regime, Phys. Rev. Lett. 114, 195003 (2015).

[16] C. Geddes et al., Compact quasi-monoenergetic photon sources from laser-plasma accelerators for nuclear detection and characterization, Nucl. Instrum. Methods Phys. Res., Sect. B 350, 116 (2015).

[17] G. A. Krafft, Spectral Distributions of Thomson-Scattered Photons from High-Intensity Pulsed Lasers, Phys. Rev. Lett. 92, 204802 (2004).

[18] D. Seipt and B. Kampfer, Nonlinear Compton scattering of ultrashort intense laser pulses, Phys. Rev. A 83, 022101 (2011).

[19] K. Krajewska and J.Z. Kaminski, Frequency scaling law for nonlinear Compton and Thomson scattering: Relevance of spin and polarization effects, Phys. Rev. A 90, 052117 (2014).

[20] L. S. Brown and T. W. B. Kibble, Interaction of intense laser beams with electrons, Phys. Rev. 133, A705 (1964).

[21] C. Brau, Oscillations in the spectrum of nonlinear Thomsonbackscattered radiation, Phys. Rev. Accel. Beams 7, 020701 (2004). 